\title{
FRACTAL FUNCTIONS AND SCHAUDER BASES
}

\author{
Z. CIESIELSKI \\ Instytut Matematyczny PAN \\ Abrahama 18, 81-825 Sopot, Poland \\ E-mail:Z.Ciesielski@impan.gda.pl
}

1. Introduction. In recent years more and more attention has been paid in mathematical papers to fractal functions and to fractal sets. There are various definitions of those objects. We assume that a compact set $K \in R^{d+1}$ is fractal, by definition, if its box (entropy) dimension $\operatorname{dim}_{b}(K) \neq j$ for $j=0,1, \ldots, d+1$ and $0<\operatorname{dim}_{b}(K)<d+1$. At the same time the function $f: I^{d} \rightarrow R^{d}, I=[0,1]$, is fractal, by definition, if its graph $\Gamma_{f}=\left\{(\boldsymbol{t}, f(\boldsymbol{t})): \boldsymbol{t} \in I^{d}\right\}$ has box dimension satisfying the inequalities $d<\operatorname{dim}_{b}\left(\Gamma_{f}\right)<d+1$. For the definitions and properties of lower $\underline{\operatorname{dim}}_{b}(K)$ and upper $\overline{\operatorname{dim}}_{b}(K)$ box (-counting) dimension we refer to $[\mathrm{F}]$. In the case $\underline{\operatorname{dim}}_{b}(K)=\overline{\operatorname{dim}}_{b}(K), \operatorname{dim}_{b}(K)$ is by definition the common value.

The relation between box dimension of the graph of a function and its Hölder exponent is known for years. In particular, it is known that the Hölder condition with some $\alpha, 0<\alpha \leq 1$, i.e.

$$
\left|f(\boldsymbol{t})-f\left(\boldsymbol{t}^{\prime}\right)\right| \leq C \cdot\left|\boldsymbol{t}-\boldsymbol{t}^{\prime}\right|^{\alpha} \quad \text { for } \quad \boldsymbol{t}, \boldsymbol{t}^{\prime} \in I^{d},
$$

implies that

$$
\operatorname{dim}_{b}\left(\Gamma_{f}\right) \leq d+1-\alpha .
$$

Our aim is to describe some subclasses of functions $f$ satisfying (1.1) for which equality takes place in (1.2). The Hölder classes, as it was shown in [C1], can be characterized by means of the coefficients of the Schauder basis expansions, and it seems natural to apply this tool to solve our problem.

In Section 2 we describe the constructions of the Schauder and Haar bases over cubes and state the main results on characterization of Hölder classes by

Lecture given at the Banach Center Colloquium on 1st April 1993. 
means of the coefficients of the Schauder and Haar expansions. Section 3 contains the main results on Hölder subclasses for which we have equality in (1.2).

2. Haar and Schauder bases. The orthogonal Haar functions over $I$, normalized in the maximum norm, can be defined by means of the function sign $(t)$. Define

$$
\begin{gathered}
h_{0}(t)=\frac{\operatorname{sign}\left(t+\frac{1}{2}\right)-\operatorname{sign}\left(t-\frac{1}{2}\right)}{2}, \\
h_{1}(t)=\frac{\operatorname{sign}\left(t+\frac{1}{2}\right)+\operatorname{sign}\left(t-\frac{1}{2}\right)}{2}-\operatorname{sign}(t) \quad \text { for } t \in R
\end{gathered}
$$

and

$$
h_{j, k}(t)=h_{1}\left(2^{k}\left(t-\frac{2 j-1}{2^{k+1}}\right)\right) \quad \text { where } \quad j=1, \ldots, 2^{k} ; \quad k=0,1, \ldots .
$$

The Haar orthogonal system on $I$ with respect to the Lebesgue measure is simply

$$
\left\{1, h_{j, k}, \quad j=1, \ldots, 2^{k} ; k=0,1, \ldots\right\} .
$$

We also note that

$$
\operatorname{supp} h_{j, k}=\left[\frac{(j-1)}{2^{k}}, \frac{j}{2^{k}}\right] .
$$

Often it is more convenient to index the Haar system as follows: $h_{1}=1$ and $h_{n}=h_{j, k}$ whenever $n=2^{k}+j$ with some $j=1, \ldots, 2^{k} ; k=0,1, \ldots$

To define the $d$-dimensional orthogonal Haar functions over $I^{d}$ properly we decompose at first the set of multi-indexes $N^{d}$, where $N=\{1,2, \ldots\}$. Using the norm $|\boldsymbol{l}|_{\infty}=\max \left(l_{1}, \ldots, l_{d}\right)$ we introduce the decompositions

$$
N^{d}=N_{-1} \cup \bigcup_{k \geq 0} N_{k} \quad \text { where } \quad N_{k}=\left\{\boldsymbol{l}: 2^{k}<|\boldsymbol{l}|_{\infty} \leq 2^{k+1}\right\}
$$

$N_{-1}$ contains $\mathbf{1}=(1, \ldots, 1)$ only and

$$
N_{k}=\bigcup_{\emptyset \neq e \subset \mathcal{D}} N_{e, k} \quad \text { with } \quad \mathcal{D}=\{1, \ldots, d\},
$$

where $N_{e, k}=\left\{\boldsymbol{l} \in N_{k}: 2^{k}<l_{i} \leq 2^{k+1}\right.$ only for $\left.i \in e\right\}$. Now, the Haar orthogonal functions over $I^{d}$ are defined as follows: $h_{\mathbf{0}}(\boldsymbol{t})=1$ and for $\boldsymbol{l} \in N_{e, k}$

$$
h_{\boldsymbol{l}}(\boldsymbol{t})=\prod_{i \in e} h_{l_{i}-2^{k}, k}\left(t_{i}\right) \prod_{i \in \mathcal{D} \backslash e}\left|h_{l_{i}, k}\left(t_{i}\right)\right| .
$$

Thus, the support of each $h_{\boldsymbol{l}}$, for $\boldsymbol{l} \in N_{e, k}$, is a dyadic cube. Actually, over $I^{d}$ we are given $2^{d}-1$ functions orthogonal to 1 , i.e. for each $e, \emptyset \neq e \subset \mathcal{D}$,

$$
h_{e}(\boldsymbol{t})=\prod_{i \in e} h_{1}\left(t_{i}\right) \prod_{i \in \mathcal{D} \backslash e} h_{0}\left(t_{i}\right)
$$


and for $\boldsymbol{l} \in N_{e, k}$

$$
h_{\boldsymbol{l}}(\boldsymbol{t})=h_{e}\left(2^{k}\left(\boldsymbol{t}-\frac{2 \boldsymbol{j}-\mathbf{1}}{2^{k+1}}\right)\right),
$$

where $j_{i}=l_{i}-2^{k}$ for $i \in e$ and $j_{i}=l_{i}$ for $i \in \mathcal{D} \backslash e$. Consequently, the support of $h_{\boldsymbol{l}}$ is the dyadic cube with center at $\frac{2 j-1}{2^{k+1}}$ and with edges of length $\frac{1}{2^{k}}$.

Below we present the graph of the typical function $h_{e}$ in case $d=2$.

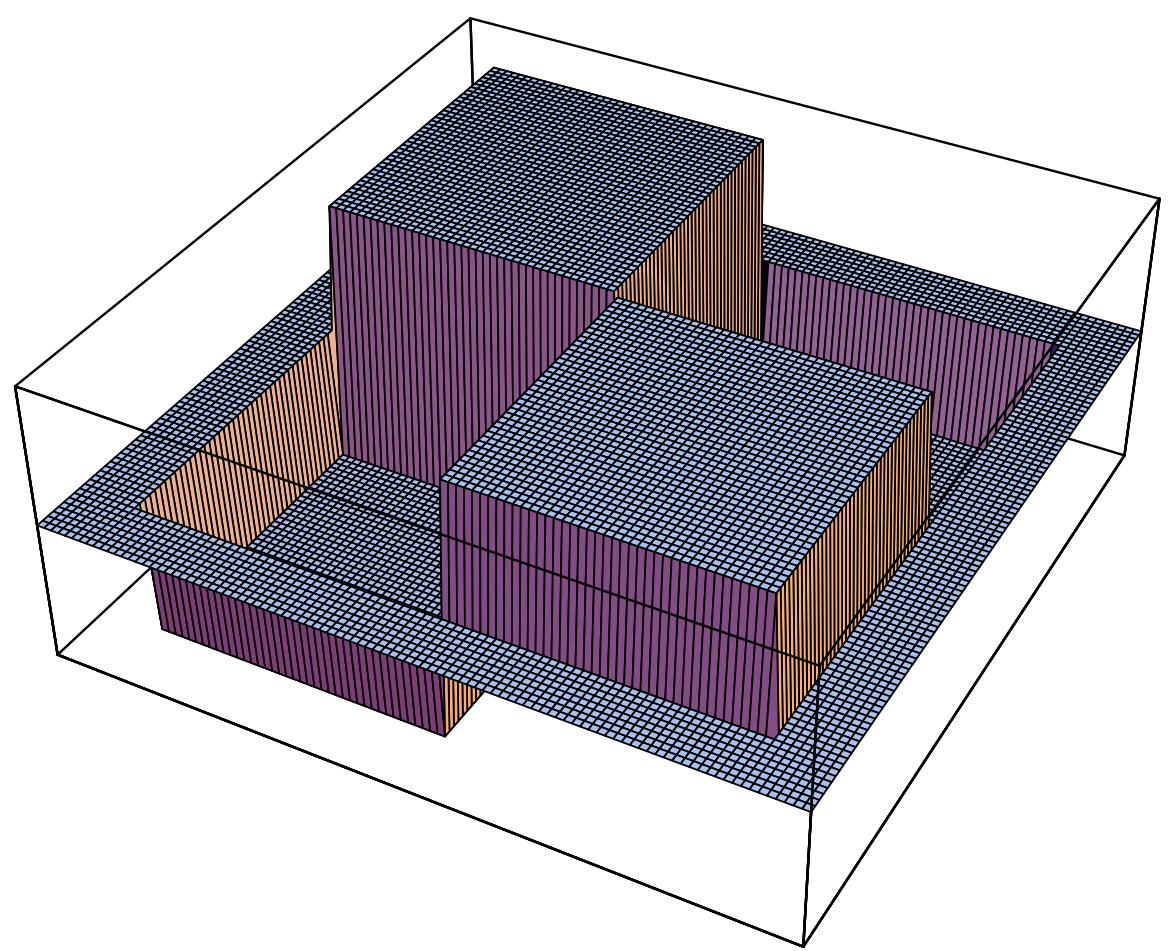

The modulus of continuity of $f \in L^{p}\left(I^{d}\right)$ in the $L^{p}$ space is defined by the formula

$$
\omega_{p}(f ; \delta)=\sup _{0<|\boldsymbol{h}|<\delta}\left(\int_{I^{d}(\boldsymbol{h})}|f(\boldsymbol{t}+\boldsymbol{h})-f(\boldsymbol{t})|^{p} d \boldsymbol{t}\right)^{1 / p}
$$

where $|\boldsymbol{h}|$ is the euclidean norm of $\boldsymbol{h}$ and $I^{d}(\boldsymbol{h})=\left\{\boldsymbol{t} \in I^{d}: \boldsymbol{t}+\boldsymbol{h} \in I^{d}\right\}$. For the later use we introduce the orthogonal projections

$$
Q_{0} f=\left(f, h_{\mathbf{0}}\right) h_{\mathbf{0}}, \quad Q_{k} f=\sum_{\boldsymbol{j} \in N_{k}} \frac{\left(f, h_{\boldsymbol{j}}\right) h_{\boldsymbol{j}}}{\left\|h_{\boldsymbol{j}}\right\|_{2}^{2}},
$$

and

$$
P_{k} f=Q_{0} f+\cdots+Q_{k} f
$$


where

$$
(f, g)=\int_{I^{d}} f(\boldsymbol{t}) g(\boldsymbol{t}) d \boldsymbol{t} \text { and }\|f\|_{p}=\left(\int_{I^{d}}|f(\boldsymbol{t})|^{p} d \boldsymbol{t}\right)^{1 / p} .
$$

It should be clear that over each dyadic cube of the $k$-th generation in $I^{d}$ the function $Q_{k} f$ is constant and equal to the mean value of $f$ over that particular dyadic cube. Thus, the Haar orthogonal system $\left\{h_{j}\right\}$ has the norms $\left\|Q_{k}\right\|_{p}, 1 \leq$ $p \leq \infty$, bounded by 1 . Consequently, the Haar system is a basis in the space $L^{p}$, $1 \leq p<\infty$. Moreover, we have

$$
\left(2^{d}-1\right)^{-1 / p} A_{k, p} \leq\left\|\sum_{\boldsymbol{n} \in N_{k}} a_{\boldsymbol{n}} \cdot h_{\boldsymbol{n}}\right\|_{p} \leq\left(2^{d}-1\right)^{1 / p^{\prime}} A_{k, p},
$$

where $1 \leq p \leq \infty, \frac{1}{p}+\frac{1}{p^{\prime}}=1, a_{\boldsymbol{n}} \in R$, and

$$
A_{k, p}=\left(\frac{1}{2^{d k}} \sum_{\boldsymbol{n} \in N_{k}}\left|a_{\boldsymbol{n}}\right|^{p}\right)^{1 / p} .
$$

Moreover, we know from [C2]

Proposition 2.3. Let $0<\alpha<\frac{1}{p} \leq 1$ and let

$$
f \sim \sum_{n \in N^{d}} a_{n} \cdot h_{n}
$$

Then

$$
\omega_{p}(f ; \delta)=O\left(\delta^{\alpha}\right) \quad \text { as } \quad \delta \rightarrow 0_{+}
$$

is equivalent to

$$
A_{k, p}=O\left(2^{-\alpha k}\right) \quad \text { as } \quad k \rightarrow \infty .
$$

Moreover, for $f \in C\left(I^{d}\right), 0<\alpha<1$, and $p=\infty$, conditions (2.4) and (2.5) are equivalent.

To define the Schauder basis over $I^{d}$ we start with the function $\psi(t)=\max [0,1-|t|]$ and the set $D$ of all dyadic points in $I$. Define $D_{0}=\{0,1\}$, $D_{k}=\left\{\frac{2 j-1}{2^{k}}: j=1, \ldots, 2^{k-1}\right\}$ and $k=1,2, \ldots$ Thus

$$
D=\bigcup_{k \geq 0} D_{k}
$$

and the Schauder functions over $I$ are defined as follows

$$
\phi_{\tau}(t)=\psi\left(2^{k}(t-\tau)\right) \quad \text { for } \quad \tau \in D_{k}, k=0,1, \ldots
$$

For the Schauder functions over $I^{d}$ it is convenient to introduce $C_{0}=D_{0}, C_{k}=$ $C_{k-1} \cup D_{k}$. Then

where

$$
C_{k}^{d}=C_{k-1}^{d} \cup D_{k, d},
$$

$$
D_{k, d}=\left\{\boldsymbol{\tau}=\left(\tau_{1}, \ldots, \tau_{d}\right) \in C_{k}^{d}: \exists_{i} \tau_{i} \in D_{k}\right\} \text { and } D_{0, d}=D_{0}^{d}
$$


Now, define

$$
\phi_{\boldsymbol{\tau}}(\boldsymbol{t})=\prod_{i \in \mathcal{D}} \psi\left(2^{k}\left(t_{i}-\tau_{i}\right)\right) \text { for } \boldsymbol{\tau} \in D_{k, d}, k=0,1, \ldots
$$

In the two dimensional case all the basic Schauder functions are obtainable, by suitable translations and rescaling, of the function presented by the picture below.

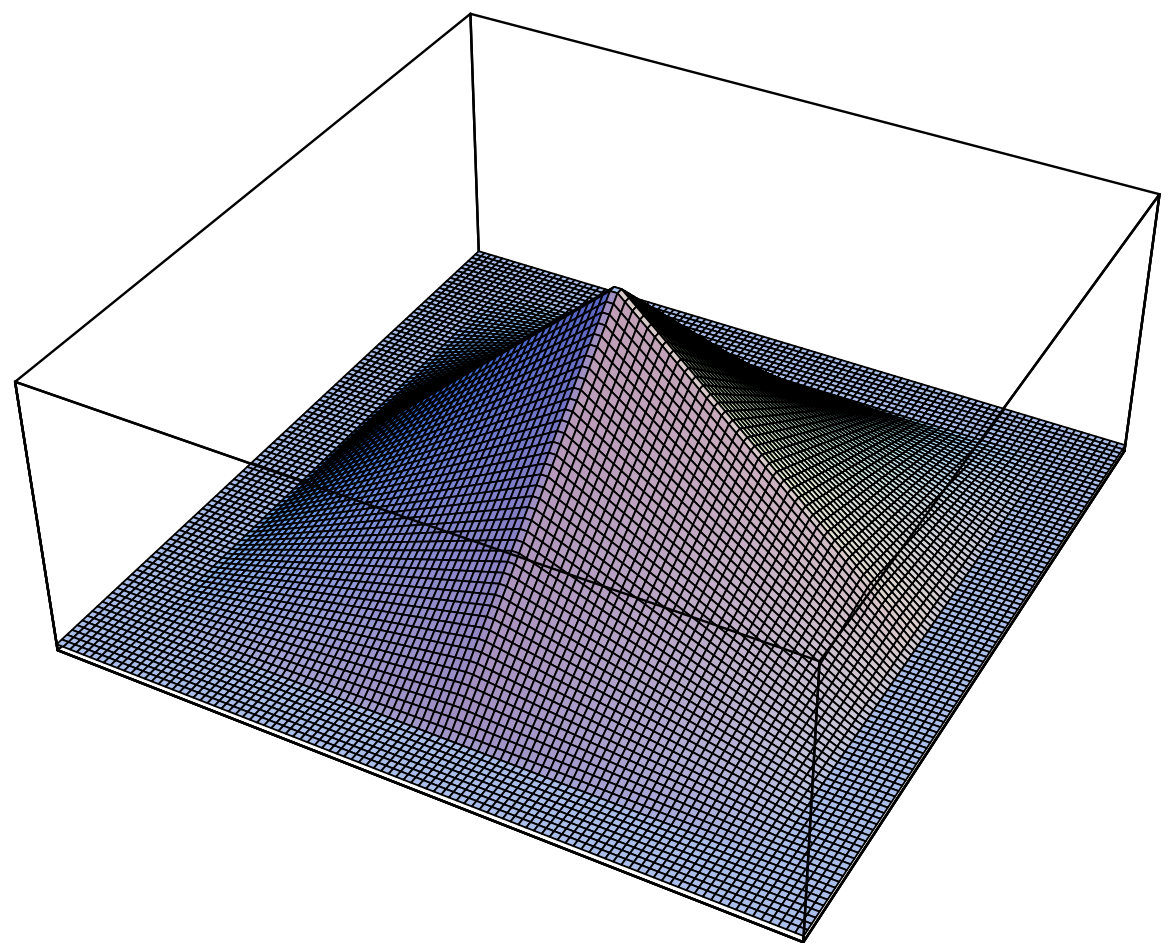

The system is called the diamond or multi-affine (cf. [R], [Se], [Sh]) basis in the Banach space $C\left(I^{d}\right)$. We mention here some of its properties. Like in the Haar case, we have with some constant $C$ depending on the dimension only, for $1 \leq p \leq \infty$, the inequalities

$$
p \cdot \frac{1}{C} \cdot B_{k, p} \leq\left\|\sum_{\boldsymbol{\tau} \in D_{k, d}} b_{\boldsymbol{\tau}} \cdot \phi_{\boldsymbol{\tau}}\right\|_{p} \leq C \cdot B_{k, p},
$$

with

$$
B_{k, p}=\left(\frac{1}{\left|D_{k, d}\right|} \sum_{\boldsymbol{\tau} \in D_{k, d}}\left|b_{\boldsymbol{\tau}}\right|^{p}\right)^{\frac{1}{p}},
$$

where $\left|D_{k, d}\right|$ is the cardinality of $D_{k, d}$.

The biorthogonal to $\left(\phi_{\boldsymbol{\tau}}(\boldsymbol{t}), \boldsymbol{\tau} \in D^{d}\right)$ system of linear functionals over $C\left(I^{d}\right)$ 
is known (see e.g. $[\mathrm{R}]$ ) and for given $f \in C\left(I^{d}\right)$ and $\boldsymbol{\tau} \in D^{d}$ the corresponding functionals are defined as follows:

$$
\begin{gathered}
b_{\boldsymbol{\tau}}(f)=f(\boldsymbol{\tau}) \quad \text { for } \quad \boldsymbol{\tau} \in D_{0, d}, \\
b_{\boldsymbol{\tau}}(f)=\frac{1}{2^{d}} \sum_{\varepsilon \in\{-1,1\}^{d}}\left(f(\boldsymbol{\tau})-f\left(\boldsymbol{\tau}^{\varepsilon}\right)\right) \quad \text { for } \quad \tau \in D_{k, d}, \quad k \geq 1
\end{gathered}
$$

where $\boldsymbol{\tau}^{\varepsilon}=\left(\boldsymbol{\tau}_{1}^{\varepsilon}, \ldots, \boldsymbol{\tau}_{d}^{\varepsilon}\right)$ with

$$
\tau_{i}^{\varepsilon}= \begin{cases}\tau_{i}+\varepsilon_{i} \cdot 2^{-k} & \text { if } \tau_{i} \in D_{k} \\ \tau_{i} & \text { if } \tau_{i} \in C_{k-1}\end{cases}
$$

It is convenient to introduce the finite dimensional projections in the space $C\left(I^{d}\right)$

$$
R_{k}(f)=\sum_{\boldsymbol{\tau} \in D_{k, d}} b_{\boldsymbol{\tau}}(f) \cdot \phi_{\boldsymbol{\tau}}
$$

The fact that $\left(\phi_{\boldsymbol{\tau}}(\boldsymbol{t}), \boldsymbol{\tau} \in D^{d}\right)$ is a Schauder basis in $C\left(I^{d}\right)$ can now be stated as follows: for each $f \in C\left(I^{d}\right)$ the series

$$
\sum_{k=0}^{\infty} R_{k}(f)
$$

converges to $f$ in the maximum norm. Finally we state the main property (cf. $[\mathrm{C} 1],[\mathrm{R}],[\mathrm{Sh}])$

Proposition 2.9. Let $0<\alpha<1, f \in C\left(I^{d}\right)$, and let

$$
f=\sum_{\tau} b_{\tau} \phi_{\tau}
$$

Then the following conditions are equivalent:

$$
\begin{gathered}
\omega_{\infty}(f ; \delta)=O\left(\delta^{\alpha}\right), \\
\max _{\boldsymbol{\tau} \in D_{k, d}}\left|b_{\boldsymbol{\tau}}\right|=O\left(2^{-\alpha k}\right), \\
\left\|f-\sum_{i \leq k} R_{i}(f)\right\|_{\infty}=O\left(2^{-\alpha k}\right) .
\end{gathered}
$$

3. Box dimension of graphs. In this section we are going to apply the Haar and Schauder bases to compute the box dimension $\operatorname{dim}_{b}\left(\Gamma_{f}\right)$ for some reasonable subclasses of the Hölder classes on cubes.

TheOREM 3.1. Let $0<\alpha \leq \beta \leq 1$ and let the function $f$ be given on $I^{d}$ by the Haar series

$$
f=\sum_{k} \sum_{\boldsymbol{n} \in N_{k}} a_{\boldsymbol{n}} \cdot h_{\boldsymbol{n}}
$$


If

$$
A_{k, \infty}=\max _{N_{k}}\left|a_{n}\right|=O\left(\frac{1}{2^{\alpha k}}\right),
$$

then

$$
\overline{\operatorname{dim}}_{b}\left(\Gamma_{f}\right) \leq d+1-\alpha .
$$

Moreover, if for large $k$ and some $C>0$,

$$
A_{k, 1}=\frac{1}{2^{k d}} \sum_{N_{k}}\left|a_{\boldsymbol{n}}\right| \geq C \cdot \frac{1}{2^{\beta k}},
$$

then

$$
\underline{\operatorname{dim}}_{b}\left(\Gamma_{f}\right) \geq d+1-\beta .
$$

COROLlary 3.2. If there is a positive finite constant $C$ such that for large $k$

$$
\frac{1}{C \cdot 2^{\beta k}} \leq \frac{1}{2^{k d}} \sum_{N_{k}}\left|a_{\boldsymbol{n}}\right| \leq \max _{N_{k}}\left|a_{\boldsymbol{n}}\right| \leq C \cdot \frac{1}{2^{\alpha k}}
$$

then

$$
d+1-\beta \leq \operatorname{dim}_{b}\left(\Gamma_{f}\right) \leq d+1-\alpha .
$$

Note, no continuity of $f$ is assumed in this statement.

Theorem 3.3. Let $0<\alpha \leq \beta \leq 1$ and let the function $f$ be given on $I^{d}$ by the Schauder series

$$
f=\sum_{k} \sum_{\boldsymbol{\tau} \in D_{k, d}} b_{\boldsymbol{\tau}} \cdot \phi_{\boldsymbol{\tau}}
$$

If

$$
B_{k, \infty}=\max _{D_{k, d}}\left|b_{\tau}\right|=O\left(\frac{1}{2^{\alpha k}}\right),
$$

then

$$
\overline{\operatorname{dim}}_{b}\left(\Gamma_{f}\right) \leq d+1-\alpha .
$$

Moreover, if for large $k$ and some $C>0$,

$$
B_{k, 1}=\frac{1}{\left|D_{k, d}\right|} \sum_{D_{k, d}}\left|b_{\boldsymbol{\tau}}\right| \geq C \cdot \frac{1}{2^{\beta k}},
$$

then

$$
\underline{\operatorname{dim}}_{b}\left(\Gamma_{f}\right) \geq d+1-\beta .
$$

Corollary 3.4. If there is a positive finite constant $C$ such that for large $k$

$$
\frac{1}{C \cdot 2^{\beta k}} \leq \frac{1}{\left|D_{k, d}\right|} \sum_{D_{k, d}}\left|b_{\boldsymbol{\tau}}\right| \leq \max _{D_{k, d}}\left|b_{\boldsymbol{\tau}}\right| \leq C \cdot \frac{1}{2^{\alpha k}},
$$

then

$$
d+1-\beta \leq \operatorname{dim}_{b}\left(\Gamma_{f}\right) \leq d+1-\alpha .
$$




\section{References}

[C1] Z. Ciesielski, On the isomorphism of the space $H_{\alpha}$ and $m$, Bull. Acad. Polon. Sci. Sér. Sci. Math. Astronom. Phys. 8 (1960), 217-222.

[C2] Z. Ciesielski, Haar orthogonal functions in analysis and probability, in: Alfred Haar Memmorial Conference (Budapest, 1985), 25-56. Colloq. Math. Soc. János Bolyai 49, North Holland 1987.

[F] K. Falconner, Fractal Geometry, Mathematical Foundations and Applications. John Wiley \& Sons Ltd. Chichester 1990.

[R] J. Ryll, Schauder bases for the space of continuous functions on an $n$-dimensional cube, Comment. Math. Prace Mat. 27 (1973), 201-213.

[Se] Z. Semadeni, Schauder Bases in Banach Spaces of Continuous Functions, Lecture Notes in Math. 918, Springer-Verlag Berlin 1982.

[Sh] A. S. Shvedov, Construction of functions by prescribing their values at binary-rational points of an m-dimensional cube. Mat. Zametki 44.2 (1988), 250-261; transl. in Math. Notes 44 (1988), 620-626. 